\title{
Conhecimento de futuros enfermeiros e médicos sobre dengue, zika e chikungunya
}

\author{
Future nurses and doctors' knowledge of dengue, zika and chikungunya \\ Conocimiento de futuros enfermeros y médicos sobre dengue, zika y chikungunya
}

\author{
Guilherme Amorim Bezerra'® ${ }^{\circledR}$; Adriani Izabel de Souza Moraes" ${ }^{\prime}$; \\ Tatiane Veteri Coneglian" @ ; Sílvia Carla da Silva André Uehara" @
}

'Hospital e Maternidade Santa Clara, Uberlândia, MG, Brasil; "Universidade Federal de São Carlos, São Carlos, SP, Brasil

\begin{abstract}
RESUMO
Objetivo: comparar o conhecimento autorreferido de formandos de medicina e enfermagem em relação à dengue, zika e chikungunya. Método: estudo transversal, exploratório e de abordagem quantitativa, realizado em uma universidade pública com 41 formandos dos cursos de enfermagem e medicina. Os dados foram coletados por meio de um questionário autorrespondido e analisados por meio de um modelo linear generalizado com distribuição binomial com função de ligação identidade. Protocolo aprovado pelo Comitê de Ética em Pesquisa. Resultados: os graduandos de medicina apresentaram as maiores médias de acertos; porém, somente as variáveis referentes ao exame clínico e à evolução e complicações apresentaram diferença estatisticamente significativa entre os cursos. Conclusão: esse estudo reforça a importância da formação de enfermeiros e médicos, para que estejam capacitados e preparados para assistir à população, desde o desenvolvimento de ações preventivas até a reabilitação de usuários acometidos por uma arbovirose.

Descritores: Infecções por Arbovírus; Educação Superior; Medicina; Enfermagem; Conhecimento.
\end{abstract}

\section{ABSTRACT}

Objective: to compare medical and nursing graduates' self-reported knowledge of dengue, zika and chikungunya. Method: this quantitative, exploratory, cross-sectional study was conducted at a public university with 41 nursing and medical graduates. Data were collected using a self-answered questionnaire and analyzed using a generalized linear model with binomial distribution and identity link function. The protocol was approved by the research ethics committee. Results: on average, medical students returned more correct answers; however, statistically significant differences were found between the courses only on variables relating to clinical examination and evolution and complications. Conclusion: this study underlines the importance of training nurses and doctors, so that they are able and prepared to provide care ranging from preventive actions through to rehabilitation of users affected by an arbovirus.

Descriptors: Arbovirus Infection; Education, Higher; Medicine; Nursing; Knowledge.

\section{RESUMEN}

Objetivo: comparar los conocimientos autoinformados de egresados de medicina y enfermería en relación con el dengue, el zika y el chikungunya. Método: estudio transversal, exploratorio con enfoque cuantitativo, realizado en una universidad pública con 41 egresados de los cursos de enfermería y medicina. Los datos se recolectaron mediante un cuestionario autoadministrado y se analizaron a través de un modelo lineal generalizado con distribución binomial con función de vínculo de identidad. Protocolo aprobado por el Comité de Ética en Investigación. Resultados: los estudiantes de medicina tuvieron el promedio más alto de respuestas correctas; sin embargo, solo las variables relacionadas con el examen clínico y la evolución y las complicaciones mostraron una diferencia estadísticamente significativa entre los cursos. Conclusión: este estudio refuerza la importancia de formar enfermeros y médicos, para que estén capacitados y preparados para atender a la población, desde el desarrollo de acciones preventivas hasta la rehabilitación de usuarios afectados por un arbovirus.

Descriptores: Infecciones por Arbovirus; Educación Superior; Medicina; Enfermería; Conocimiento.

\section{INTRODUÇÃO}

Dengue, zika e chikungunya são doenças infecciosas transmitidas pelo Aedes aegypti (A. aegypti). Essas enfermidades estão incluídas na Lista Nacional de Notificação Compulsória de Doenças, Agravos e Eventos de Saúde Pública, possuindo grande relevância para estudos, em especial a dengue, por ser endêmica em algumas regiões brasileiras, além de possuir elevadas taxas de morbidade ${ }^{1}$.

O Brasil presenciou a emergência de arbovírus com o Zika Vírus (ZIKV) em 2015; e, Chikungunya Vírus (CHKV) em 2014, além de epidemias frequentes de dengue ${ }^{2}$. A disseminação das arboviroses, ao longo do tempo, tem sido facilitada por fatores como crescimento das populações nos grandes centros urbanos, transporte entre regiões distantes, alterações climáticas e dificuldade para controlar de forma efetiva a ocorrência de surtos ${ }^{3}$.

Estudo financiado por bolsa da Fundação de Apoio à Pesquisa de São Paulo (FAPESP), Processo 2017/07764-7. Recebido apoio da Coordenação de Aperfeiçoamento de Pessoal de Nível Superior (CAPES, Brasil).

Autora correspondente: Tatiane Veteri Coneglian. E-mail: tatiane_veteri@hotmail.com

Editora Científica: Cristiane Helena Gallasch; Editora Associada Helena Maria Scherlowski Leal David 
Em 2019, no Brasil, foram registrados 1.668 .680 casos prováveis de dengue, zika e chikungunya, dos quais 852 resultaram em óbito ${ }^{4}$. Não obstante o fato de compartilharem semelhanças epidemiológicas, apresentando ciclo de transmissão basicamente em áreas urbanas, a evolução e complicações decorrentes da infeç̧ão por essas arboviroses seguem cursos distintos ${ }^{4}$. Assim, o diagnóstico clínico e laboratorial é dificultado, especialmente, na presença da cocirculação de mais de um tipo de arbovírus em uma mesma localidade ${ }^{5}$.

A compreensão dos agentes de saúde sobre as particularidades das arboviroses influencia na detecção precoce da infecção e no diagnóstico prematuro de um quadro clínico grave da doença ${ }^{6}$. Entretanto, estudos têm apontado deficiências no conhecimento de profissionais de saúde, em especial médicos ${ }^{7}$ e enfermeiros ${ }^{8}$ e de estudantes dos cursos de enfermagem ${ }^{9}$ e medicina ${ }^{10}$ sobre aspectos epidemiológicos e fisiopatológicos da dengue, zika e chikungunya, além de dificuldades no reconhecimento e diferenciação dos sinais e sintomas ocasionados por esses arbovírus, mesmo em áreas endêmicas.

Tais estudos apresentam uma lacuna no conhecimento, pois não avaliaram conjuntamente todos os aspectos inerentes à dengue, zika e chikungunya, como etiologia, ciclo de transmissão, fisiopatologia, epidemiologia, sinais e sintomas, diagnóstico, tratamento e meios prevenção, além de não realizarem uma comparação entre os conhecimentos apresentados pelos formandos dos cursos de medicina e enfermagem, sendo assim, esta pesquisa visa minimizar essa lacuna do conhecimento.

O controle, prevenção, diagnóstico e tratamento efetivo dessas arboviroses estão estreitamente relacionados com a capacitação técnica dos profissionais de saúde, em especial enfermeiros e médicos inseridos nos serviços da Atenção Primária à Saúde (APS) e pronto atendimento. Nesse contexto, o conhecimento adquirido pelos enfermeiros e médicos durante a formação acadêmica pode ser considerado um dos principais pilares de sustentação dos serviços de saúde, além de ser um fator decisório para uma assistência de qualidade à comunidade. Dessa forma, este estudo questionase qual o nível de conhecimento dos formandos em enfermagem e medicina em relação a dengue, zika e chikungunya?

O conhecimento dos formandos de enfermagem e medicina sobre as arboviroses constitui-se em uma das tarefas a serem cumpridas por aqueles que estão envolvidos com o encaminhamento das políticas públicas de saúde, particularmente, no que concerne ao ensino de graduação em enfermagem e medicina. Ressalta-se a necessidade de realizar uma análise comparativa entre os subgrupos, uma vez que são profissões distintas, mas que exercem um trabalho interdependente, assim, possíveis deficiências no conhecimento de uma classe profissional pode influenciar a assistência de outra categoria.

Nesse contexto, este estudo teve como objetivo comparar o conhecimento autorreferido de formandos de medicina e enfermagem em relação à dengue, zika e chikungunya.

\section{MÉTODO}

Estudo transversal, exploratório e de abordagem quantitativa, desenvolvido em uma universidade pública do interior de São Paulo, com dados coletados no período de fevereiro a maio de 2019.

A população inicialmente definida foi de 30 formandos de enfermagem e 40 de medicina, que corresponde ao número de alunos que ingressam anualmente nesses cursos.

Entretanto, os acadêmicos concluintes dos cursos de enfermagem e medicina variam e normalmente não correspondem ao número de ingressantes; em 2019, 20 estudantes concluíram o curso de enfermagem e 35 o curso de medicina, compondo a população deste estudo. Contudo, dois estudantes de enfermagem se recusaram a participar e um foi excluído do estudo por ser membro da pesquisa.

Para o curso de medicina, houve quatro recusas e sete foram excluídos após cinco tentativas para realizar a coleta de dados. Por fim, a amostra foi constituída por 17 acadêmicos do último ano de enfermagem e 24 formandos de medicina de uma Instituição Pública de Ensino Superior (IPES).

Para a inclusão dos participantes foi considerado o seguinte critério: ser acadêmico do último ano de graduação em enfermagem ou medicina de uma IPES do interior de São Paulo. Foram excluídos acadêmicos após cinco tentativas sem sucesso para realizar a coleta de dados e ser participante da pesquisa. Os dados foram coletados por meio de um questionário autorrespondido, que foi construído com base nos Protocolos do Ministério da Saúde sobre dengue, zika e chikungunya ${ }^{5,11,12}$

O questionário foi composto por questões agrupadas em blocos referentes ao exame clínico, evolução e complicação continham questões sobre os sinais e sintomas das doenças; os blocos sobre exame clínico e laboratoriais abordavam questões referentes aos diagnósticos das doenças; ainda, os blocos que incluíam exame clínico e evolução e complicação também versavam sobre questões relativas ao tratamento das arboviroses. 
Os dados coletados foram inseridos em um banco de dados construído no Programa Microsoft Excel ${ }^{\circledR}$. As variáveis foram codificadas conforme as alternativas de cada item, para viabilizar a análise estatística. Para comparar média de acerto entre os cursos foi proposto um modelo linear generalizado com distribuição binomial com função de ligação identidade. Tal modelo foi escolhido devido à natureza das variáveis de desfecho, número de eventos (acertos) em um total de ensaios (questões).

A classe de modelos lineares generalizados é uma extensão do modelo linear tradicional, o qual permite que a média populacional seja dependente de um preditor linear por meio de uma função de ligação não linear e permite que a distribuição de probabilidade da variável resposta seja qualquer membro da família exponencial (Distribuição Normal, Binomial, Poisson e Gama) ${ }^{13}$.

Todos os gráficos apresentados foram feitos com o auxílio do software R, versão 3.4.1 e as análises, por meio do SAS 9.2. Para todas as comparações adotou-se um nível de significância de $5 \%$ e intervalo de confiança de $95 \%$. 0 protocolo de pesquisa foi aprovado pelo Comitê de Ética em Pesquisa da Universidade em que foi realizado o estudo.

\section{RESULTADOS}

Os dados relacionados aos acertos nas questões sobre dengue, zika e chikungunya são apresentados na Tabela 1.

TABELA 1: Média de acertos nas questões sobre dengue, zika e chikungunya, segundo formandos do curso de enfermagem e medicina. São Carlos, SP, Brasil, 2019.

\begin{tabular}{|c|c|c|c|c|}
\hline \multirow{2}{*}{ Variáveis } & \multicolumn{2}{|c|}{ Enfermagem $(n=17)$} & \multicolumn{2}{|c|}{ Medicina ( $n=24)$} \\
\hline & Média & Desvio Padrão & Média & Desvio Padrão \\
\hline \multicolumn{5}{|l|}{ Fisiopatologia } \\
\hline Dengue & 75,74 & 13,60 & 80,21 & 9,69 \\
\hline Zika & 53,78 & 13,86 & 64,29 & 15,19 \\
\hline Chikungunya & 55,15 & 18,25 & 64,06 & 17,02 \\
\hline \multicolumn{5}{|l|}{ Epidemiologia } \\
\hline Dengue & 74,21 & 11,52 & 74,04 & 10,59 \\
\hline Zika & 79,14 & 18,68 & 74,62 & 11,37 \\
\hline Chikungunya & 77,06 & 16,49 & 72,08 & 14,74 \\
\hline \multicolumn{5}{|l|}{ Exame clínico } \\
\hline Dengue & 67,16 & 16,26 & 90,63 & 14,18 \\
\hline Zika & 57,52 & 26,71 & 78,70 & 19,36 \\
\hline Chikungunya & 58,24 & 24,04 & 84,17 & 16,92 \\
\hline \multicolumn{5}{|c|}{ Exame laboratorial } \\
\hline Dengue & 55,88 & 25,81 & 69,79 & 16,45 \\
\hline Zika & 49,41 & 22,49 & 56,67 & 21,80 \\
\hline Chikungunya & 42,65 & 19,29 & 51,04 & 21,47 \\
\hline \multicolumn{5}{|c|}{ Evoluções e complicações } \\
\hline Dengue & 62,75 & 18,19 & 75,69 & 12,98 \\
\hline Zika & 74,51 & 17,79 & 89,58 & 10,78 \\
\hline Chikungunya & 64,71 & 26,27 & 78,47 & 18,04 \\
\hline \multicolumn{5}{|c|}{ Meios de prevenção } \\
\hline Dengue & 71,43 & 25,25 & 75,60 & 18,59 \\
\hline Zika & 52,94 & 29,78 & 63,10 & 24,89 \\
\hline Chikungunya & 53,78 & 29,25 & 61,90 & 26,53 \\
\hline \multicolumn{5}{|l|}{ Total } \\
\hline Dengue & 69,53 & 8,17 & 79,08 & 5,96 \\
\hline Zika & 62,88 & 10,50 & 72,04 & 7,06 \\
\hline Chikungunya & 60,65 & 13,92 & 70,74 & 8,86 \\
\hline
\end{tabular}

A média total de acertos para dengue, zika e chikungunya, respectivamente, foi de 79,72 e 70,7 para medicina e de 69,5, 62,8 e 60,6 para enfermagem.

Os acadêmicos de medicina apresentaram as maiores médias de acertos quando comparados aos acadêmicos de enfermagem em todas as variáveis, com exceção da variável referente à epidemiologia das arboviroses, em que as 
médias de acertos entre os formandos em medicina foram de 74, 74,6 e 72 para dengue, zika e chikungunya, respectivamente, e, para os estudantes de enfermagem de 74,2 para dengue, 79,1 para zika e 77 para chikungunya.

Destacam-se as médias de acertos em relação ao exame clínico, que para medicina foram de 90,6, 78,7 e 84,1, respectivamente para dengue, zika e chikungunya, enquanto para a enfermagem foram de 67,1, 57,5 e 58,2, respectivamente. 0 bloco de varáveis referentes ao exame clínico apresentaram as maiores diferenças nas médias de acertos entre os cursos, indicando maior discrepância nos níveis de conhecimento dos estudantes.

O resultado geral mostrou uma diferença estatisticamente significativa ao comparar o conhecimento entre os formandos de medicina e de enfermagem para dengue, zika e chikungunya (Tabela 2).

TABELA 2: Comparação das médias de acerto entre os cursos de enfermagem e medicina sobre dengue, zika e chikungunya. São Carlos, SP, Brasil, 2019.

\begin{tabular}{|c|c|c|c|}
\hline Variáveis & Estimativa & Intervalo de Confiança (\%) & Valor $p$ \\
\hline \multicolumn{4}{|l|}{ Fisiopatologia } \\
\hline Dengue & $-4,47$ & $-13,62-4,67$ & 0,340 \\
\hline Zika & $-10,5$ & $-22,03-1,02$ & 0,070 \\
\hline Chikungunya & $-8,92$ & $-19,68-1,85$ & 0,100 \\
\hline \multicolumn{4}{|l|}{ Epidemiologia } \\
\hline Dengue & 0,17 & $-7,38-7,72$ & 0,960 \\
\hline Zika & 4,52 & $-3,32-12,36$ & 0,260 \\
\hline Chikungunya & 4,98 & $-3,52-13,47$ & 0,250 \\
\hline \multicolumn{4}{|l|}{ Exame clínico } \\
\hline Dengue & $-23,47$ & $-30,74--16,2$ & $<0,010$ \\
\hline Zika & $-21,19$ & $-30,74--11,64$ & $<0,010$ \\
\hline Chikungunya & $-25,93$ & $-34,67--17,2$ & $<0,010$ \\
\hline \multicolumn{4}{|c|}{ Exames laboratoriais } \\
\hline Dengue & $-13,91$ & $-28,86-1,05$ & 0,070 \\
\hline Zika & $-7,25$ & $-21,1-6,59$ & 0,300 \\
\hline Chikungunya & $-8,39$ & $-23,83-7,04$ & 0,290 \\
\hline \multicolumn{4}{|c|}{ Evolução e complicações } \\
\hline Dengue & $-12,95$ & $-24,66--1,24$ & 0,030 \\
\hline Zika & $-15,07$ & $-24,89--5,25$ & $<0,010$ \\
\hline Chikungunya & $-13,77$ & $-25,22--2,32$ & 0,020 \\
\hline \multicolumn{4}{|c|}{ Meios de prevenção } \\
\hline Dengue & $-4,17$ & $-14,56-6,23$ & 0,430 \\
\hline Zika & $-10,15$ & $-21,72-1,41$ & 0,090 \\
\hline Chikungunya & $-8,12$ & $-19,71-3,46$ & 0,170 \\
\hline \multicolumn{4}{|l|}{ Total } \\
\hline Dengue & $-9,55$ & $-13,41--5,7$ & $<0,010$ \\
\hline Zika & $-9,16$ & $-13,51--4,82$ & $<0,010$ \\
\hline Chikungunya & $-10,09$ & $-14,49--5,69$ & $<0,010$ \\
\hline
\end{tabular}

Apesar de os estudantes de enfermagem terem obtido a maior média de acerto no que se refere aos aspectos epidemiológicos das arboviroses, tal fato não representa uma diferença estatisticamente significativa.

Destaca-se que foi observada diferença estatisticamente significativa $(p<0,05)$ entre os cursos de medicina e enfermagem apenas para as variáveis relacionadas ao exame clínico e à evolução e complicações, em que os formandos de medicina obtiveram a maior média de acerto.

A Figura 1 demonstra a comparação entre as proporções de acertos entre os grupos de formando de enfermagem e de medicina, sobre o exame clínico para as três arboviroses estudadas.

Os formandos de medicina obtiveram uma proporção de acerto que variou de 70 a $100 \%$ quando questionados sobre o exame clínico da dengue, sendo que $2 / 3$ acertaram mais de $90 \%$. Já entre os formandos em enfermagem, a variação da proporção de acerto referente ao exame clínico da dengue foi de 60 a quase $80 \%$ e metade dos participantes acertaram entre 60 e $70 \%$. 

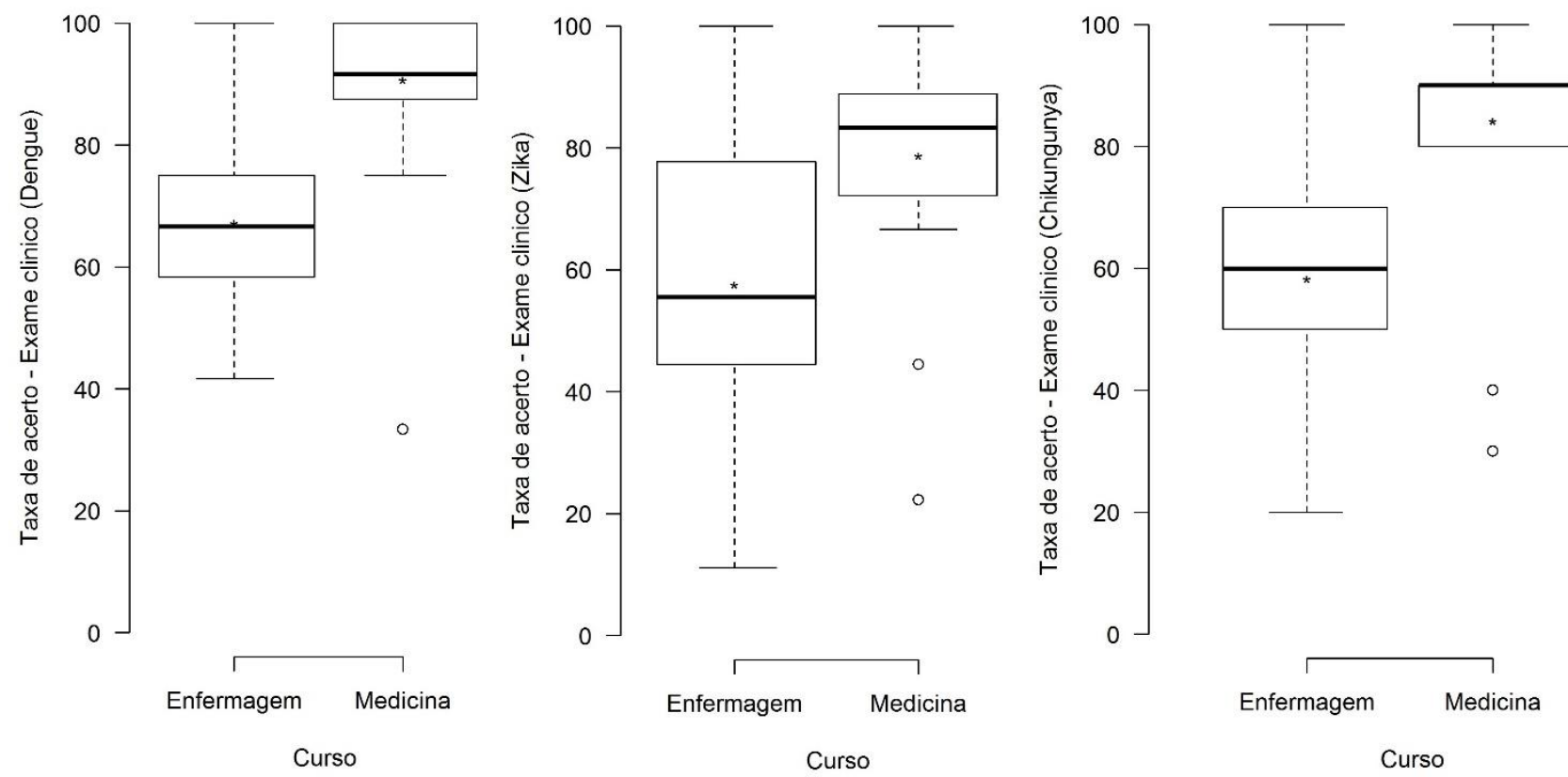

FIGURA 1: Comparação entre as proporções de acertos entre os formandos de enfermagem e medicina sobre o exame clínico da dengue, zika e chikungunya. São Carlos, SP, Brasil, 2019.

Em relação ao exame clínico da zika, a proporção de acerto da enfermagem variou de $40 \%$ a quase $80 \%, 2 / 3$ dos participantes acertaram mais que $50 \%$; enquanto para a medicina a variação de acerto foi de quase $80 \%$ a $90 \%$ e $1 / 3$ acertaram mais de $80 \%$.

Os concluintes de enfermagem obtiveram uma proporção de acerto variando de $50 \%$ a $70 \%$ sobre o exame clínico da chikungunya e a metade dos participantes acertaram 60\%; enquanto os formandos de medicina mantiveram desempenho superior, com uma proporção de acerto que variou de $80 \%$ a $90 \%$.

Quanto à evolução das doenças citadas, a Figura 2 demonstra a comparação entre as proporções de acertos entre os formandos de enfermagem e medicina.
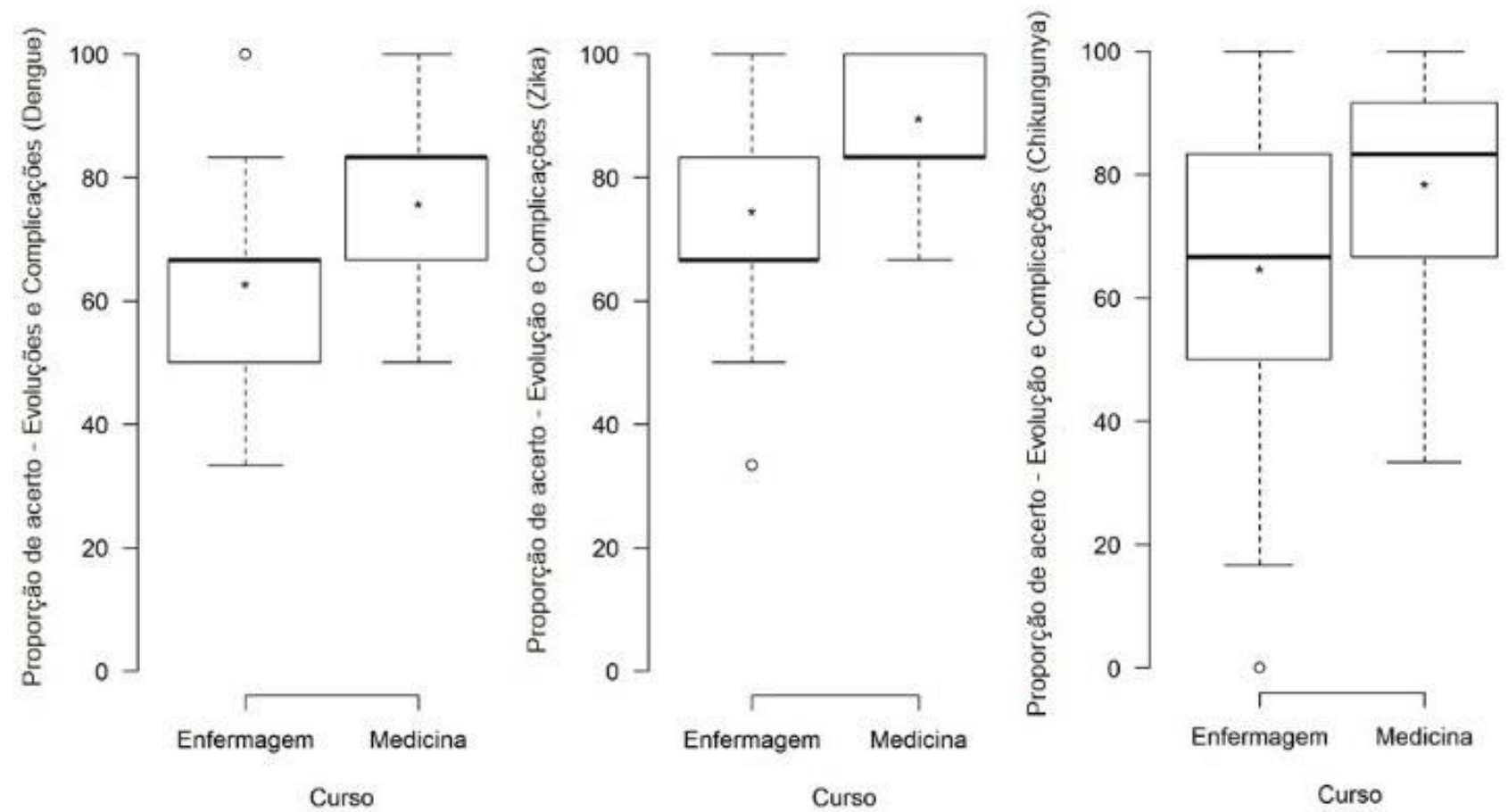

FIGURA 2: Comparação entre as proporções de acertos entre os formandos de enfermagem e medicina sobre evolução e complicações da dengue, zika e chikungunya. São Carlos, SP, Brasil, 2019. 
Os concluintes de medicina obtiveram uma proporção de acerto de $75,69 \%$ no que se refere à evolução e complicações para dengue. Já entre os formandos de enfermagem, a proporção de acerto sobre a evolução e complicações da dengue foi de $62,75 \%$.

Quanto à zika a proporção de acerto para evolução e complicações foi de 74,51\% para enfermagem; e de 89,58\% para a medicina.

Os formandos de enfermagem obtiveram uma proporção de acerto de $64,71 \%$ para a evolução e complicações da chikungunya e os formandos de medicina $78,47 \%$.

\section{DISCUSSÃO}

O conhecimento aprofundado de formandos de medicina e enfermagem sobre a reprodução do vetor, transmissão dos vírus, mecanismos fisiopatológicos das arboviroses, além do diagnóstico, tratamento e meios de prevenção são fundamentais no atual contexto, uma vez que o Brasil é considerado um país endêmico para essas doenças.

A carência de conhecimento sobre as arboviroses e suas especificidades aliado à dificuldade de evitar sua ocorrência ou tratar as complicações representa um desafio para o controle dessas doenças. Destaca-se a execução de ações preventivas de exposição aos vetores, sobretudo por meio de práticas educativas desenvolvidas nos serviços da APS; além de enfatizar o papel do enfermeiro como principal agente de educação em saúde ${ }^{14}$.

O nível de conhecimento apresentado pelos estudantes de enfermagem sobre os meios de prevenção das arboviroses sinaliza a necessidade de melhor qualificação, em especial no que se refere à zika e chikungunya. As atividades de ensino supervisionadas em campo prático são importantes ferramentas que contribuem para a formação, colaborando para que os estudantes se tornem aptos a compor e liderar equipes multidisciplinares, bem como fomentar a prática interprofissional e colaborativa.

Estudo realizado sobre a percepção de risco e o nível de conhecimento sobre as doenças transmitidas pelo $A$. aegypti mostrou que os médicos apresentaram melhor desempenho quando comparados com os enfermeiros ${ }^{15}$. Tal fato pode ser explicado, em parte, pela maior carga horária exigida para a formação de médicos, entre graduação e residência; portanto, maior possibilidade de dedicação a temáticas específicas do currículo.

O fato de os enfermeiros serem responsáveis por funções assistenciais e gerenciais nos diferentes níveis de atenção à saúde pode comprometer a qualidade da atenção, pois ao longo da graduação, a abordagem a determinadas temáticas pode ser considerada negligenciada, bem como insuficiente para o aprendizado e aplicação prática.

Pesquisa mostrou que 87,5\% (70) dos graduandos de enfermagem possuíam conhecimentos insatisfatórios sobre a dengue ${ }^{13}$. De forma semelhante, um estudo revelou que dos 313 estudantes de ciências da saúde, apenas $21 \%$ apresentaram um bom conhecimento da dengue ${ }^{16}$. O fato de a dengue ser considerada uma doença recorrente e, frequentemente, se apresentar de forma benigna, pode contribuir para que os futuros enfermeiros direcionem o foco dos estudos para patologias de maior gravidade.

Estudo avaliou o conhecimento de 500 estudantes universitários de quatro cursos, incluindo enfermagem e medicina, sobre a zika e revelou que $62,8 \%$ (314) dos participantes possuíam pouco conhecimento sobre a doença. Os estudantes de medicina obtiveram o melhor desempenho geral e os de enfermagem, o pior ${ }^{10}$. A dificuldade para diferenciar os sinais e sintomas, que também podem se manifestar em outras doenças febris, aliada ao fato da zika ser alvo de maior atenção da mídia no Brasil e dos profissionais de saúde apenas recentemente, são também possíveis explicações para essa lacuna no conhecimento.

Em relação à chikungunya, pesquisa realizada em 2017, envolvendo 563 profissionais e estudantes da área da saúde constatou que 43,3\% (244) dos participantes não tinham conhecimento sobre a existência do vírus e, daqueles que estavam cientes, apenas $31 \%$ revelaram um conhecimento adequado sobre a doença ${ }^{9}$. Esses achados explicitam a urgência em oferecer capacitação e treinamento, tanto para os profissionais diretamente envolvidos no cuidado aos pacientes, como para docentes dos cursos de graduação da área da saúde nas instituições de ensino superior. Ressaltase que tanto a chikungunya como a dengue e zika são doenças com elevado potencial de causar epidemias, comprometendo o bem-estar da população e onerando os serviços de saúde.

Os enfermeiros são os agentes de saúde que mantém maior proximidade com os usuários dos serviços de saúde, sendo considerados os principais responsáveis pelo cuidado e ações de educação em saúde. Esses profissionais são os principais atores na APS que em articulação com os serviços da vigilância em saúde desenvolvem atividades para a prevenção de arboviroses, visando minimizar os riscos de surtos e epidemias ${ }^{17}$. 
As atividades educativas sobre a prevenção de arboviroses direcionadas aos usuários e à comunidade geralmente são desenvolvidas e organizadas por enfermeiros. Esses profissionais também prestam assistência direta às pessoas com diagnóstico de uma dessas doenças, exigindo conhecimentos teóricos consolidados sobre todos os aspectos inerentes às arboviroses.

A relevância do exame clínico da dengue torna-se ainda mais acentuada ao considerar que o critério clínicoepidemiológico é o mais indicado para confirmar os casos suspeitos de dengue em período epidêmico, enquanto o critério laboratorial exerce papel auxiliar ${ }^{5}$.

Profissionais com conhecimentos insuficientes sobre as características clínicas da doença podem retardar a tomada de decisão relacionada às medidas de contenção da disseminação do vírus e prejudicar o manejo clínico adequado. Além dos conhecimentos técnicos relacionados ao diagnóstico, terapia medicamentosa, solicitação e interpretação de exames, os profissionais de saúde devem estar familiarizados com a epidemiologia dessas doenças.

A falta de conhecimento adequado sobre a epidemiologia pode levar esses profissionais a orientarem inadequadamente os pacientes, comprometendo o sucesso das estratégias de controle da transmissão do vírus. Estudo avaliou os conhecimentos, atitudes e práticas de 79 profissionais de saúde sobre a infecção pela dengue, sendo $82,0 \%$ médicos, e foram identificadas lacunas significativas no que diz respeito à epidemiologia e prevenção ${ }^{7}$.

Esses achados refletem o tradicional modelo biologicista de assistência à saúde, predominante por muito tempo na saúde pública, centrado apenas no médico e doença, sem considerar a relevância do contexto e dos determinantes sociais do processo saúde-doença ${ }^{18}$.

As características epidemiológicas das arboviroses sinalizam a necessidade de intensificar as ações de combate ao vetor e direcionar as ações de prevenção ${ }^{19}$. O cuidado integral à saúde requer que médicos, além das práticas específicas de sua área de atuação também desenvolvam, durante a consulta, ações de educação em saúde. Da mesma forma, o enfermeiro deve possuir conhecimento técnico adequado para reconhecer os sinais e sintomas das doenças, uma vez que realiza o acolhimento e a classificação de risco.

Destaca-se, a necessidade de capacitar os profissionais de saúde já atuantes para lidar com a nova realidade de doenças emergentes como zika e chikungunya, refletindo na formação de enfermeiros e médicos, pois em muitos serviços de saúde, esses profissionais realizam preceptoria de estudantes, além de acompanhar a realização dos estágios.

Pesquisas realizadas com estudantes e profissionais de enfermagem revelaram deficiências no conhecimento em relação a vários aspectos sobre a zika e chikungunya ${ }^{10,13,16}$. A deficiência no ensino teórico e prático das arboviroses faz com que médicos e enfermeiros ao iniciarem suas atividades profissionais, sintam-se pouco confiantes e enfrentem dificuldades para identificar e tratar essas doenças. Enfatiza-se a necessidade do aprimoramento do conhecimento antes mesmo da inserção dos profissionais no mercado de trabalho ${ }^{20}$.

Diante do exposto, fica evidente a necessidade de repensar as estratégias de ensino, priorizando a formação de profissionais com conhecimento teórico robusto e vivência prática. Esses profissionais devem ser capazes de articular os diferentes saberes, sobretudo, no que diz respeito à prevenção, diagnóstico e tratamento das doenças transmitidas pelo A. aegypti.

\section{Limitações do estudo}

Esse estudo teve como limitação a resistência dos graduandos, especialmente do curso de medicina, para participação no estudo, o que dificultou a coleta dos dados. Também, a carência de estudos, especialmente, nacionais que viabilizassem uma comparação com os resultados obtidos neste estudo.

\section{CONCLUSÃO}

Conclui-se que entre os formandos do curso de medicina e do curso de enfermagem, as médias relacionadas ao exame clínico foram as únicas com diferença estatísticamente significativa. No entanto, os resultados das demais variáveis demonstraram a necessidade de desenvolver estratégias que favoreçam uma formação profissional multidisciplinar que contemple aspectos de promoção, proteção, prevenção, recuperação e reabilitação da saúde.

\section{REFERÊNCIAS}

1. Marcondes CB, Contigiani M, Gleiser RM. Emergent and reemergent arboviruses in south America and the Caribbean: why so many and why now? J Med Entomol [Internet]. 2017 [cited 2020 Jan 13]; 54(3):509-32. DOI: https://doi.org/10.1093/jme/tjw209.

2. Lima-Camara TN. Emerging arboviruses and public health challenges in Brazil. Rev Saúde Pública [Internet]. 2016 [cited 2020 Jan 13]; 50:36. DOI: https://doi.org/10.1590/S1518-8787.2016050006791. 
3. Vogels CBF, Rcükert C, Cavany SM, Perkins TA, Ebel GD, Grubaugh ND. Arbovirus coinfection and co-transmission: a neglected public health concern? PLoS Biol. [Internet]. 2019 [cited 2020 Jan 13]; 17(1):e3000130. DOI: https://doi.org/10.1371/journal.pbio.3000130.

4. Ministério da Saúde (BR). Secretaria de Vigilância em Saúde. Boletim epidemiológico [Internet]. 2019 [cited 2020 Jan 13 ]. Available from: http://portalarquivos2.saude.gov.br/images/pdf/2019/dezembro/23/Boletim-epidemiologico-SVS-38-2interativo.pdf.

5. Ministério da Saúde (BR). Secretaria de Vigilância em Saúde. Departamento de Vigilância das Doenças Transmissíveis. Dengue: diagnóstico e manejo clínico: adulto e criança. 5. ed. Brasília: Ministério da Saúde, 2016.

6. Department of Public Health of New York State. Arboviral (Arthropod-borne viral) diseases. [Internet]. 2017 [cited 2018 Nov 10]. Available from: https://www.health.ny.gov/diseases/communicable/arboviral/fact_sheet.htm.

7. Handel AS, Ayala EB, Borbor-Cordova MJ, Fessler AG, Finkelstein JL, Espinoza RXR, Ryan SJ, Stewart-lbarra AM. Knowledge, attitudes and practices regarding dengue infection among public sector healthcare providers in Machala, Ecuador. Trop Dis Travel Med Vaccines [Internet]. 2016 [cited 2020 Jan 13]; 2(8). DOI: https://doi.org/10.1186/s40794-016-0024-y.

8. Kageguka DC, Desrochers RE, Mwangi R, Mgabo MR, Alifrangis M, Kavishe RA, Mosha FW, Kulkarni MA. Knowledge and practice regarding dengue and chikungunya: a cross-sectional study among healthcare workers and community in Northern Tanzania. Trop Med Int Health [Internet]. 2017 [cited 2020 Jan 13]; 22(5):583-93. DOI: https://doi.org/10.1111/tmi.12863.

9. Mallhi TH, Khan YB, Tanveer N, Bukhsh A, Khan AH, Aftab RA, Khan OH, Khan TM. Awareness and knowledge of chikungunya infection following its outbreak in Pakistan among health care students and professionals: a nationwide survey. Peer J. [Internet]. 2018 [cited 2020 Jan 13]; (6):e5481. DOI: https://doi.org/10.7717/peerj.5481.

10. Rabbani SA, Mustafa F, Shouquair T, Mohamad I, Tahsin N. Zika vírus disease knowledge among the future health-care providers of the United Arab Emirates. J Adv Pharm Technol Res. [Internet]. 2018 [cited 2020 Jan 13]; 9(1):20-5. DOI: https://doi.org/10.4103/japtr.JAPTR_239_17.

11. Ministério da Saúde (BR). Secretaria de Vigilância em Saúde. Departamento de Vigilância das Doenças Transmissíveis. Chikungunya: manejo clínico / Ministério da Saúde, Secretaria de Vigilância em Saúde, Departamento de Vigilância das Doenças Transmissíveis. - Brasília: Ministério da Saúde, 2017. 65 p.: il.

12. Ministério da Saúde (BR). Secretaria de Vigilância em Saúde. Vírus Zika no Brasil: a resposta do SUS [recurso eletrônico] / Ministério da Saúde, Secretaria de Vigilância em Saúde. - Brasília: Ministério da Saúde, 2017.136 p. il.

13. McCullagh, P, Nelder JA. Generalized Linear Models. 2nd. Edition. Chapman and Hall, London, 1989.

14. Liang G, Gao X, Gould EA. Factors responsible for the emergence of arboviroses; strategies, challenges and limitations for their control. Emerg Microbes Infect. [Internet]. 2015 [cited 2020 Jan 13]; 4(3):e18. DOI: https://doi.org/10.1038/emi.2015.18.

15. Menchaca-Armenta I, Ocampo-Torres M, Hernández-Gómez A, Zamora-Cerritos K. Risk perception and level of knowledge of diseases transmitted by Aedes aegypti. Rev Inst Med Trop São Paulo [Internet]. 2018 [cited 2020 Jan 13]; (60):e10. DOI: http://dx.doi.org/10.1590/s1678-9946201860010.

16. Choi JS, Kim KM. Infection-control knowledge, atitude, pactice, and risk perception of occupational exposure to zika virus among nursing students in Korea: a cross-sectional survey. J Infect Public Health [Internet]. 2018 [cited 2020 Jan 13]; 11:840-4. DOI: http://dx.doi.org/10.1590/s1678-9946201860010.

17. Kumar JR, Kishore A, Kumar SD, Shamshul A, Govind D, Sangharshila B, Phoolgen S, Gayatri K. Knowledge and Awareness regarding dengue among the undergraduate health science students of dengue hit region of Nepal. Int Res. J. Medical Sci. [Internet]. 2016 [cited 2020 Jan 13]; 4(1):8-12.

18. Al Awfi MS, Al Sharabi BA, Al Alimi A, Abkar MA. Knowledge, atitudes and practices regarding dengue fever in a cohort of nursing students. JAMMR. [Internet]. 2019 [cited 2020 Jan 13]; 29(3):1-9. DOI: https://doi.org/10.9734/jammr/2019/v29i330073.

19. Moore KS. Assessing nurse practitioner knowledge of zika virus. J Nurse Pract [Internet]. 2016 [cited 2020 Jan 13]; 12(10). DOI: http://dx.doi.org/10.1016/j.nurpra.2016.09.012.

20. Saringe S, Kajeguka DC, Kagirwa DD, Mgabo MR, Emidi B. Healthcare workers knowledge and diagnostic practices: a need for dengue and chikungunya training in Moschi Municipality, Kilimanjaro Tanzania. BMC Res Notes [Internet]. 2019 [cited 2020 Jan 13]; 12(1):43. DOI: https://doi.org/10.1186/s13104-019-4074-x. 